Publications of the Astronomical Society of the Pacific, 115:170-177, 2003 February

(C) 2003. The Astronomical Society of the Pacific. All rights reserved. Printed in U.S.A.

\title{
Molecular Hydrogen in the Ring Nebula: Clumpy Photodissociation Regions
}

\author{
Angela K. Speck ${ }^{1}$ And Margaret Meixner ${ }^{2}$ \\ Astronomy Department, University of Illinois at Urbana-Champaign, 1002 West Green Street, Urbana, IL 61801; speckan@missouri.edu \\ AND \\ George H. Jacoby and Patricia M. KnezeK \\ WIYN Observatory, P.O. Box 26732, Tucson, AZ 85726 \\ Received 2002 August 15; accepted 2002 October 11
}

\begin{abstract}
We present a 0"65 resolution $\mathrm{H}_{2} 1-0 S(1) 2.122 \mu \mathrm{m}$ image of the Ring Nebula (NGC 6720), which was taken with the Near Infrared Imager at the WIYN $3.5 \mathrm{~m}$ telescope on Kitt Peak. The high resolution of the $\mathrm{H}_{2}$ observation is sufficient to reveal the finer structure of the molecular material in this nebula. The morphology of the molecular emission is compared to that of the ionized emission from the Ring Nebula as seen by the Hubble Space Telescope (HST; He II, [O III], and [N II]), and it is clear that the dark clumps seen by $H S T$ match the locations of clumpy $\mathrm{H}_{2}$ emission, suggesting that these clumps are similar to the cometary knots seen in the Helix Nebula. As with the Helix, the clumpy $\mathrm{H}_{2}$ emission from the main ring of the Ring Nebula is contained within the optically bright ionized nebula, implying that the molecular gas is shielded inside dense condensations. Comparison of the observed $\mathrm{H}_{2}$ average surface brightnesses for the Ring Nebula [(1.5 \pm $0.5) \times 10^{-4} \mathrm{ergs} \mathrm{cm}^{-2} \mathrm{~s}^{-1} \mathrm{sr}^{-1}$ ] with time-dependent models of the expected $\mathrm{H}_{2}$ emission from planetary nebulae (PNe) shows that it is consistent with $\mathrm{H}_{2}$ excitation in photodissociation regions (PDRs), confirming previous suggestions. Comparison of the Ring Nebula $\mathrm{H}_{2}$ emission with a younger PN, NGC 2346, and an older PN, the Helix Nebula, suggests an evolution in $\mathrm{H}_{2}$ surface brightness consistent with the time-dependent PDR models. Moreover, the knots of molecular gas appear to become more isolated as the PN evolves, consistent with optical studies of knots in PNe.
\end{abstract}

\section{INTRODUCTION}

The classical paradigm for the structure of planetary nebulae $(\mathrm{PNe})$ is that of an ionized gas bubble bounded by neutral gas and molecules. Only a fraction of the PNe observed exhibit molecular emission (e.g., Zuckerman \& Gatley 1988; Huggins \& Healy 1989). However, when molecular gas is detected in a PN, it exists within ionized regions, leading to a reevaluation of the classic structure. In the Helix Nebula (NGC 7293), dense condensations (cometary knots) are known to exist in the main ionized nebula (e.g., O’Dell \& Handron 1996; Meaburn et al. 1998). These cometary knots have been shown to contain molecular material by imaging them in the CO $J=1-0(2.6 \mathrm{~mm})$ line and the $\mathrm{H}_{2}$ 1-0 S(1) $2.12 \mu \mathrm{m}$ line (Huggins et al. 2002; Speck et al. 2002).

Historically, the interpretation of the $\mathrm{H}_{2} 2.12 \mu \mathrm{m}$ line emission from PNe has been controversial. Some papers claim that the line arises from shocked-gas regions resulting from the interaction of the fast central star wind with the slowly

\footnotetext{
${ }^{1}$ Current address: Physics and Astronomy Department, University of Missouri, Columbia, MO 65211.

${ }^{2}$ Current address: Space Telescope Science Institute, 3700 San Martin Drive, Baltimore, MD 21218.
}

expanding nebula (e.g., Beckwith, Gatley, \& Persson 1978; Zuckerman \& Gatley 1988). Others claim that it is more passively excited by the far-ultraviolet (FUV) and X-ray radiation of the central star in photodissociation regions (PDRs; e.g., Latter et al. 2000; Vicini et al. 1999; Speck et al. 2002; see also Latter et al. 1995). For molecular gas in clumps within the ionized region of a nebula, the surface of the clumps will constitute PDRs, where the ionized gas meets the molecular gas. In this case, we would expect there to be a thin layer of ionized gas emission on the surface of the clump facing the central star, and the $\mathrm{H}_{2}$ emission would lie behind the ionized gas emission as seen from the central star. Thus, a detailed comparison of the $\mathrm{H}_{2}$ emission with the ionized gas emission can help to establish the case for PDRs. Such a study has been done for the Helix Nebula (Huggins et al. 2002), and with higher angular resolution we can extend such studies to more distant and younger PNe.

Another PN in which molecular emission appears to originate from within the ionized nebula is the Ring Nebula (NGC 6720, M57; e.g., Latter et al. 1995). The Ring Nebula was included in the Hubble Space Telescope (HST) WFPC2 study of O'Dell et al. (2002), who showed knots in five PNe, suggesting that these knots are common and that there is some 
evolution to them. In order to understand how these knots contribute to PN evolution and to establish origin of the $\mathrm{H}_{2}$ emission, we have taken a high angular resolution ( $\left.\sim 0^{\prime \prime} 65\right)$, well-sampled image of the molecular emission from the Ring Nebula.

In $\S 2$, we present the new high-resolution observations of molecular hydrogen in the Ring Nebula; $§ 3$ compares the new observations to previous studies of the ionized gas and discusses qualitatively the implications of the relative distributions of the molecular and ionized gas; $\S 4$ argues that the $\mathrm{H}_{2}$ emission in the Ring Nebula arises from PDRs, not shocks, and discusses the evolution of the $\mathrm{H}_{2}$ emission in PNe in the context of the time-dependent PDR models of Natta \& Hollenbach (1998, hereafter NH98) and how this relates to the observed evolution of knots in the ionized gas. The conclusions are summarized in $\S 5$.

\section{OBSERVATIONS}

We have obtained two images of the Ring Nebula in the near-infrared (NIR) $2.122 \mu \mathrm{m} v=1-0 S(1)$ line of molecular hydrogen $\left(\mathrm{H}_{2}\right)$ taken using the Near-Infrared Imager camera (NIRIM; Meixner, Young Owl, \& Leach 1999) at two different telescopes: the WIYN $3.5 \mathrm{~m}$ telescope on Kitt Peak on the night of 2001 September 3 and the Mount Laguna $1 \mathrm{~m}$ telescope on the nights of 1999 June 15-16.

At the WIYN $3.5 \mathrm{~m}$ telescope, the imager was used with the 0 "'.17 pixel $^{-1}$ plate scale, giving the $256 \times 256$ pixel array an imaging area of 43 ".5 $\times 43$ ".5. The angular size of the Ring Nebula (semimajor axis $\sim 40^{\prime \prime}$ ) demanded mosaic mapping. Off positions were taken regularly at offsets of $5^{\prime}$ from the central star. Each frame had an integration time of $50 \mathrm{~s}$ and was imaged several times. The final mosaicked image represents 500-700 s of total integration time, with the longest times found in the central regions.

At the Mount Laguna telescope, the imager was used with the $1^{\prime \prime}$ pixel $^{-1}$ plate scale, giving an imaging area of $256^{\prime \prime} \times$ $256^{\prime \prime}$. In this case, the whole nebula fits in a single frame. The final image was composed of five $200 \mathrm{~s}$ exposures.

The data were reduced and compiled using IRAF. ${ }^{3}$ Each exposure was flat-fielded to eliminate large pixel-to-pixel sensitivity variations in the detector array and was then skysubtracted. Sky emission maps were constructed by taking the average of several sky frames obtained close to the target field in both time and space. We used point sources in each field as reference points to determine relative offsets between frames. Finally, the frames were co-added using the derived offsets. The resulting high-resolution $\mathrm{H}_{2}$ image from WIYN is shown in Figure 1, compared to the HST (Bond et al. 1998) [N II] image. The resolution is seeing-limited at $\sim 0$ ". 65 .

\footnotetext{
${ }^{3}$ IRAF is distributed by the National Optical Astronomy Observatory, which is operated by the Association of Universities for Research in Astronomy, Inc., under cooperative agreement with the National Science Foundation.
}

The WIYN image covers only the main optically bright ring. The Mount Laguna image (not shown) shows both the ring and the outer $\left(\sim 2^{\prime}\right.$ radius $)$ circularly symmetric $\mathrm{H}_{2}$ emission together with the intermediate flower-petal structure previously reported by Kastner et al. (1994). These images show that the large-scale $\mathrm{H}_{2}$ emission morphology matches that of the $\mathrm{H} \alpha$ as seen by Balick et al. (1992).

Previous studies of the $\mathrm{H}_{2}$ emission from the Ring Nebula have defined the average surface brightness in a variety of different ways. Here the average surface brightness for both images was calculated by taking the average over several rectangular blocks that cover the bright $\mathrm{H}_{2}$ region and then taking the average of these values. By using this method, we do not cover as many dark regions, and thus our average surface brightness is somewhat higher than previously quoted $(\sim 7 \times$ $10^{-5}$ ergs $\mathrm{cm}^{-2} \mathrm{~s}^{-1} \mathrm{sr}^{-1}$; e.g., Zuckerman \& Gatley 1988; Kastner et al. 1994). Applying our method for calculating the average surface brightness to the image from Kastner et al. (1994), we find that the value for the surface brightness is $50 \%$ higher than quoted in Kastner et al. (1994). In order to compare with previous observations of this and other nebulae, it is necessary to be sure that methods for calculating average surface brightnesses are consistent.

In the case of the WIYN observations, calibration was done using SAO 048300, FS 07, and FS 31 as standard stars, which were observed in $\mathrm{H}_{2}$ and the $K^{\prime}$ band before and after the mosaic mapping. The $K$-band fluxes for these standard stars were obtained from Hunt et al. (1998). The flux calibration gives a peak value for the $\mathrm{H}_{2}$ emission of $\sim 6 \times 10^{-4} \mathrm{ergs} \mathrm{cm}^{-2} \mathrm{~s}^{-1} \mathrm{sr}^{-1}$. The average brightness is $\sim 1.9 \times 10^{-4} \mathrm{ergs} \mathrm{cm}^{-2} \mathrm{~s}^{-1} \mathrm{sr}^{-1}$.

For the Mount Laguna image, the calibration used standard stars HD 129653 and HD 161903. The $K$-band fluxes for these standard stars were obtained from Elias et al. (1982). The flux calibration gives a peak value for the $\mathrm{H}_{2}$ emission of $\sim 4 \times 10^{-4}$ ergs $\mathrm{cm}^{-2} \mathrm{~s}^{-1} \mathrm{sr}^{-1}$. The average brightness is $\sim 1.5 \times 10^{-4} \mathrm{ergs} \mathrm{cm}^{-2} \mathrm{~s}^{-1} \mathrm{sr}^{-1}$. The difference in the peak brightness can be attributed to the difference in resolution (the Mount Laguna image has seeing of 2 ".8).

The average surface brightness from the bright central ring of the Ring Nebula is $\sim 1.5( \pm 0.5) \times 10^{-4}$ ergs $\mathrm{cm}^{-2} \mathrm{~s}^{-1} \mathrm{sr}^{-1}$. This is the average of the brightnesses from both our observations and that of Kastner et al. (1994).

\section{COMPARISON WITH PREVIOUS OBSERVATIONS}

The earliest map of the molecular emission from the Ring Nebula was an image of the $\mathrm{H}_{2}$ emission with a resolution of $\sim 10^{\prime \prime}$ (Beckwith et al. 1978). Beckwith et al. (1978) described their image as a smooth ringlike distribution. However, they showed that the $\mathrm{H}_{2}$ emission is spatially coincident with that of $\left[\mathrm{O}_{\mathrm{I}}\right]$, indicative of high-density neutral clumps within the ionized region. This was the first observation to suggest that the molecular material in the Ring Nebula is contained within the ionized zone. The atomic emission arises from the transition 


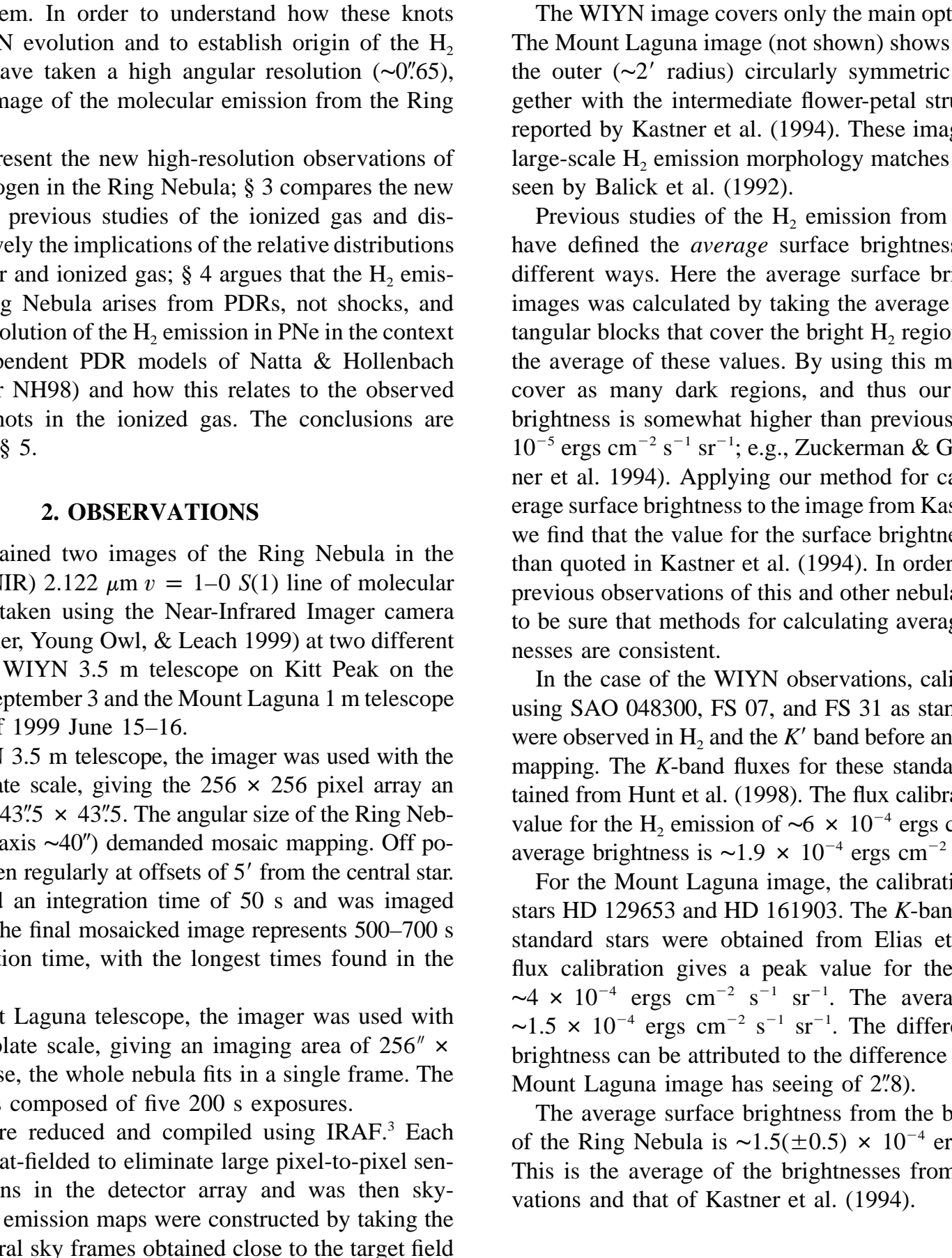

Fig. 1.-Comparison of the distribution of emission from $\mathrm{H}_{2}$ and [N II]. Top panel: Ring Nebula in the $\mathrm{H}_{2} v=1-0 S(1) 2.122 \mu \mathrm{m}$ line taken at the WIYN $3.5 \mathrm{~m}$ telescope. Bottom panel: HST [N II] image (Bond et al. 1998).

zone between the dense molecular clumps and the ionized medium. This result was confirmed by Greenhouse, Hayward, \& Thronson (1988), who compared the molecular hydrogen emission distribution with that of atomic hydrogen $(\mathrm{H} \mathrm{r})$. They found that the $\mathrm{H}_{2}$ and $\mathrm{H}$ I distributions are coincident and again indicative of clumps of molecular material embedded in the ionized nebula. They also pointed out that at $\sim 10^{\prime \prime}$ resolution, the
$H_{2}$ image looks clumpier than the lower resolution (19".6) $H_{2}$ image of Zuckerman \& Gatley (1988) and suggested that, as even higher resolutions are achieved, the apparent clumpiness of the molecular emission will increase. Indeed, the higher resolution images of Kastner et al. (1994; seeing limited at 2") and Latter et al. (1995; detector limited at $\sim 1^{\prime \prime}$ ) show an increased clumpiness in the $\mathrm{H}_{2}$ emission. Latter et al. (1995) also 
observed filamentary structures that radiate away from knots of $\mathrm{H}_{2}$ emission in the main ring. The clumpy nature of the molecular gas in the Ring Nebula and its large-scale spatial coincidence with the ionized nebula are also borne out by observations of CO emission (Huggins \& Healy 1986; Bachiller et al. 1989).

Our $\mathrm{H}_{2}$ image of the Ring Nebula ( 0"65 resolution) reveals the finer structure of the clumps, not resolved by previous images, and allows a detailed comparison with the HST images, which have 0.11 resolution. Comparison of the $\mathrm{H}_{2}$ image with [N II], a low-energy ionized species (Fig. 1), shows that the distribution of molecular gas is identical to that of the lowest energy ionized gas, except that the molecular emission is clumpier. The coincidence of the $[\mathrm{N} \mathrm{II}]$ emission with the $\mathrm{H}_{2}$ emission implies that the molecular gas is somehow shielded from the ionizing radiation by being inside denser (more optically thick) knots that are surrounded by PDRs where the surfaces of the molecular knots meet the ionized gas.

Comparing the high resolution $\mathrm{H}_{2}$ images with the Hubble Heritage image of the Ring Nebula (Bond et al. 1998; Fig. 2), it is clear that the dark clumps seen by HST match the locations of clumpy $\mathrm{H}_{2}$ emission, suggesting that these clumps are similar to the cometary knots seen in the Helix Nebula (e.g., O'Dell \& Handron 1996). Indeed, the morphology of $\mathrm{H}_{2}$ emission from the Ring Nebula shows a remarkable resemblance to that of the Helix Nebula (see Speck et al. 2002). As with the Helix, the clumpy $\mathrm{H}_{2}$ structure of the Ring Nebula may imply either that these knots are remnants of denser condensations from the asymptotic giant branch phase (e.g., Dyson et al. 1989) or that they formed through instabilities caused by the passing of either the ionization front (e.g., Capriotti 1971) or the fast-wind shock front (Vishniac 1994). Indeed, the observations of $\mathrm{H} \alpha$ from the outer halo of the Ring Nebula suggest that the main ionization front has long since passed through the main ring (Balick et al. 1992), although smaller ionization fronts still exist at the surface of the dense knots.

Knots and filaments seen in the ionized gas images of PNe are further discussed by O'Dell et al. (2002), who studied five different nearby PNe and compared the nature of the knots and filaments in these different objects. They found that there is a progression in the appearance of these knots that correlates with the age (evolutionary status) of the nebulae, starting with knots closest to the central star having dark tangential structures that are not aligned with the central star and are located near the main ionization front. These structures do not have bright edges. In a slightly older PN, the structures are still dark (no bright edges) but now show well-defined knots and have some indications of tails (e.g., the Ring Nebula). Finally, the knots are very well defined with bright, photoionized edges facing the central star and tails radially oriented away from the central star (e.g., the Helix Nebula).

A closer comparison of the Ring Nebula $\mathrm{H}_{2}$ emission with that of the Helix Nebula supports the idea of evolution of the $\mathrm{H}_{2}$ emission in PNe. The $\mathrm{H}_{2}$ emission knots are more isolated in the Helix Nebula than in the Ring Nebula, suggesting that a longer evolution has allowed more erosion of the molecular gas.

\section{THE ORIGIN AND EVOLUTION OF THE MOLECULAR HYDROGEN EMISSION IN PLANETARY NEBULAE}

NH98 have demonstrated that previous PDR models severely underestimate the expected $\mathrm{H}_{2}$ flux in $\mathrm{PNe}$ because they ignore $\mathrm{X}$-rays. By including time-dependent effects expected from the evolution of the PN and the X-ray emission from the central star in their PDR models, NH98 have reproduced the high $\mathrm{H}_{2}$ surface brightnesses observed in evolved PNe. Once the central star temperature exceeds $10^{5} \mathrm{~K}$, the soft X-rays heat and ionize the neutral gas to a much higher degree than can the FUV stellar flux alone. This results in stronger $2 \mu \mathrm{m} \mathrm{H}_{2}$ emission as these lines are temperature sensitive. The $\mathrm{X}$-ray flux maintains a high level of $\mathrm{H}_{2}$ emission to much later evolutionary stages of PNe. Shock excitation of the $\mathrm{H}_{2}$ molecules is also included in the NH98 models but was shown to be negligible compared to the PDR emission.

The models of NH98 incorporate shells, tori, or clumps of molecular material by using a filling factor given by the solid angle the molecular region subtends as seen from the central star. They also suggest that extreme clumpiness, as seen in the Helix Nebula, would produce further enhancement of the $\mathrm{H}_{2}$ emission in the PDR. Thus, the models of NH98 are applicable to a wide range of nebular morphologies, including the "bowtie" morphology often associated with $\mathrm{H}_{2}$ emission (Zuckerman \& Gatley 1998; Kastner et al. 1996), and to inhomogeneous nebulae.

The models of NH98 have been successfully applied to the young bow-tie planetary nebula NGC 2346 by Vicini et al. (1999). Vicini et al. modeled NGC 2346 assuming that the molecular gas forms a clumpy torus around the central star.

NGC 2346 is believed to be very similar in structure to the Ring Nebula (Latter et al. 1995). Therefore, we compare our observations of the Ring Nebula to the models of NH98. NH98 found that the surface brightness in the $\mathrm{H}_{2} v=1-0 \mathrm{~S}(1)$ line can be as high as $10^{-5}$ to $10^{-4}$ ergs $\mathrm{cm}^{-2} \mathrm{~s}^{-1} \mathrm{sr}^{-1}$, even after $10^{4} \mathrm{yr}$, consistent with our observed surface brightness of $\sim 1.5( \pm 0.5) \times 10^{-4} \mathrm{ergs} \mathrm{cm}^{-2} \mathrm{~s}^{-1} \mathrm{sr}^{-1}$. Thus, the high level of $\mathrm{H}_{2}$ emission seen in the Ring Nebula is consistent with that expected from models of thermal $\mathrm{H}_{2}$ excitation in PDRs. These PDRs originate at the surfaces of the many molecular knots that give the nebula its clumpy nature.

The NH98 models also predict that the brightness of the $\mathrm{H}_{2}$ emission in PNe evolves along with the nebula. Therefore, we might expect to see a difference in the $\mathrm{H}_{2}$ emission from $\mathrm{PNe}$ of different ages. Hence, we compare observed values of the $\mathrm{H}_{2}$ 1-0 S(1) surface brightness for the Ring Nebula, the Helix Nebula, and NGC 2346 to one of the NH98 models that was tailored to the PN, NGC 2346 (model 1 of Vicini et al. 1999; 

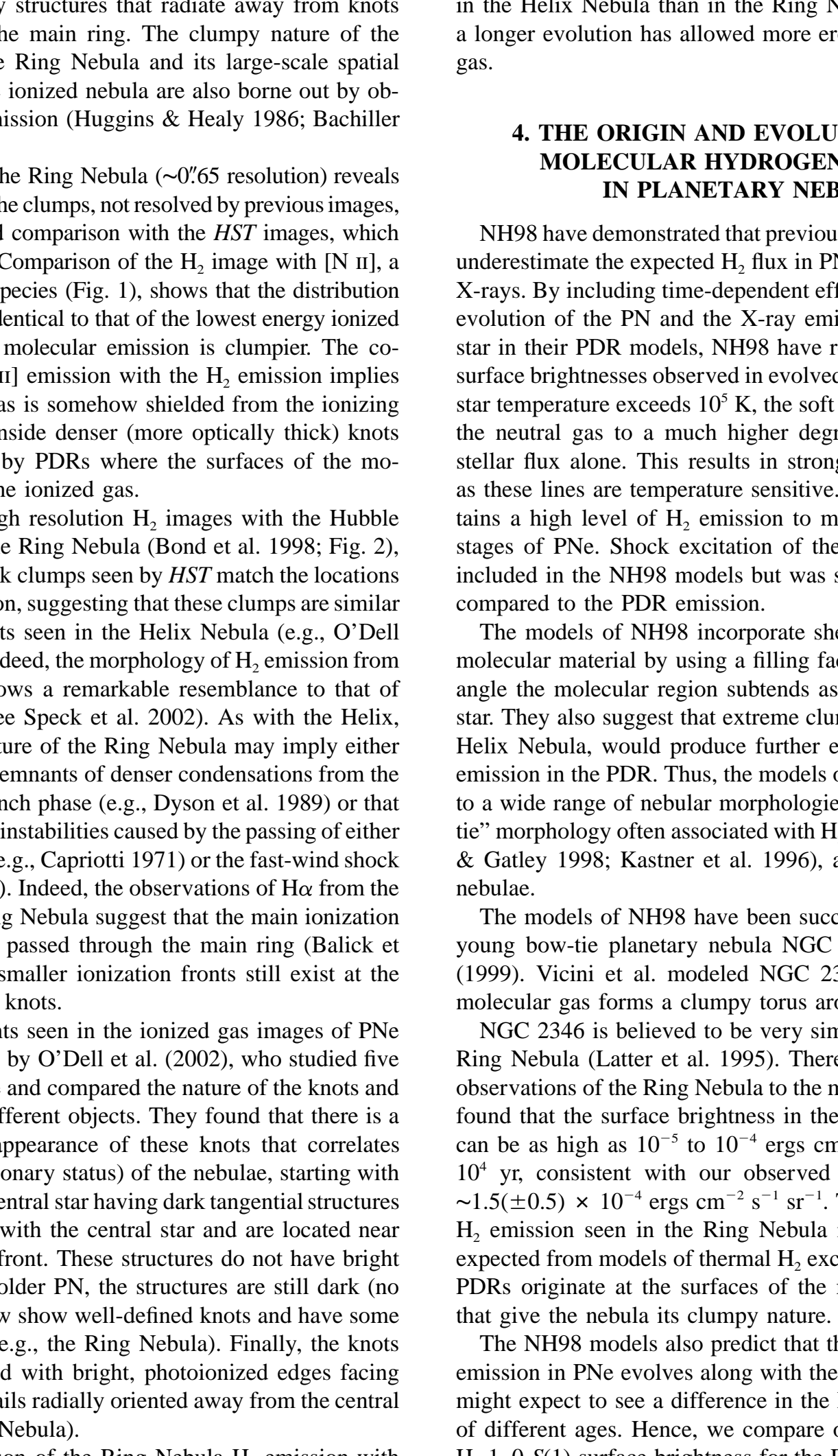

FIG. 2.-Comparison of the Hubble Heritage image with the $\mathrm{H}_{2}$ image. Top panel: Hubble Heritage image of the Ring Nebula in He II, [O III] and [N II]. Bottom panel: Ring Nebula in the $\mathrm{H}_{2}$. The letters refer to common features. 


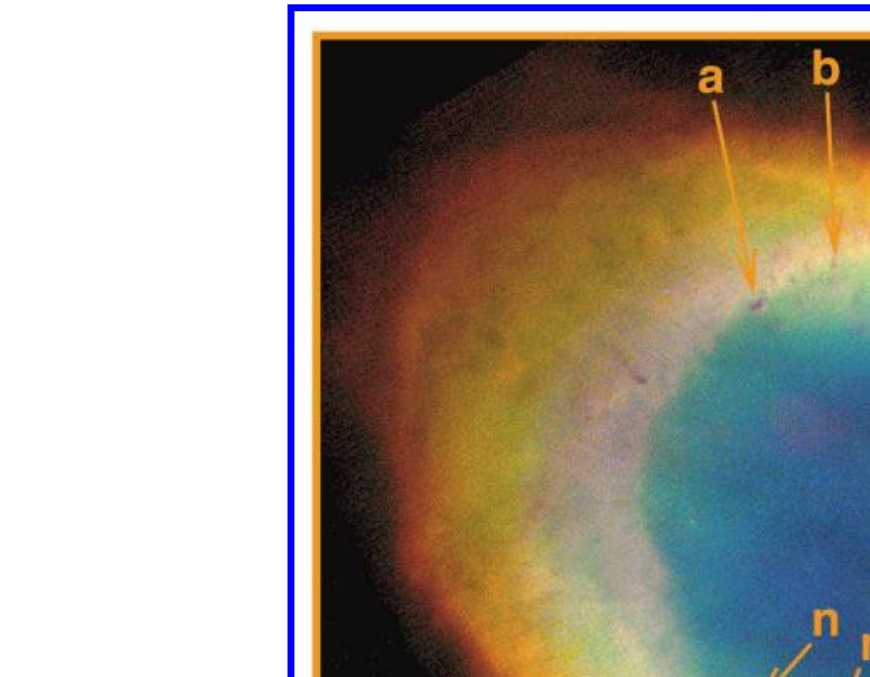

FIG. 3.-Comparison of observed $\mathrm{H}_{2} v=1-0 S(1)$ surface brightnesses for NGC 6720, NGC 2346 (Vicini et al. 1999), and NGC 7293 (Speck et al. 2002) with model predictions of model 1 from Vicini et al. (1999) represented by the dashed line. The model shows how the $\mathrm{H}_{2}$ intensity changes over the lifetime of a PN.

see Fig. 3). The Ring Nebula and NGC 2346 are believed to be very similar in structure (e.g., Latter et al. 1995) and have central stars of similar mass (NGC 2346: $M_{\text {core }}=0.7 M_{\odot}[\mathrm{Vi}-$ cini et al. 1999]; Ring: $M_{\text {core }}=0.69 M_{\odot}$ [Galli et al. 1997]). The Helix Nebula is included because the morphology of the molecular hydrogen emission is remarkably similar for both the Helix Nebula and the Ring Nebula. The important parameters of these nebulae can be found in Table 1 .

In order to examine how the evolutionary status of $\mathrm{PNe}$ relates to $\mathrm{H}_{2}$ emission, we need to know the age of the nebulae. The dynamical ages for both the Ring Nebula and Helix Nebula were found by O'Dell et al. (2002) to be $1500 \pm 225$ and $10,600_{-1200}^{+2300} \mathrm{yr}$, respectively. These ages are based on direct measurements of the inner radius of the expanding ring of the main nebula and on the expansion velocities from the ionized lines. It is not possible to apply this method to NGC 2346, as its inner torus is viewed edge-on. Vicini et al. (1999) found a dynamical age for this object by measuring the distance between the peaks in the edge-on torus. However, this method does not measure the inner radius; rather, it measures the average radius of the main bright "ring." Furthermore, their expansion velocity comes from observations of the molecular emission. The expansion velocity of a PN varies for different ionized and molecular species (e.g., Bryce, Balick, \& Meaburn 1994; Balick et al. 1992). Therefore, in order to compare like with like, we have applied the same approach to the Ring and Helix Nebulae, and thus the ages are older than $\left(3000_{-1700}^{+2500}\right.$ and $19,000_{-8000}^{+11,000} \mathrm{yr}$, respectively) but within error of O'Dell et al. (2002). Another factor that contributes to the uncertainty in the dynamical age of these nebulae is the distance. For the Ring Nebula, quoted distances vary from 400 to 800 pc (e.g., O'Dell et al. 2002; Zhang 1993; Acker et al. 1992; Pier et al. 1993; Gathier 1987). We have chosen to use the trigonometric parallax distance of $500 \pm 200 \mathrm{pc}$ from Pier et al. (1993), which is within the error of the expansion distance of $450 \mathrm{pc}$ derived by O'Dell et al. (2002).

Our observations of the Ring Nebula and Helix Nebula agree well with the NGC 2346 model of Vicini et al. (1999), given some of the differences between the PNe. NGC 2346 is the youngest of the three PN (at $2500 \mathrm{yr}$; Vicini et al. 1999) and has the lowest intensity and, by design, fits the models the best. The $\mathrm{H}_{2}$ emission arises from collisionally excited $\mathrm{H}_{2}$ gas warmed by the soft X-rays of the central star. The Ring Nebula also fits very well with the prediction from the model. This is expected since the Ring has a very similar core mass to NGC 2346 and these two nebulae are believed to have very similar structures (Latter et al. 1995). The dynamical age of the Ring, $\sim 3000 \mathrm{yr}$, places it at the rising edge of the model curve's $\mathrm{H}_{2}$ $1-0 S(1)$ surface brightness. This rising edge predicts that the Ring's $\mathrm{H}_{2} 1-0 S(1)$ surface brightness may be expected to

TABLE 1

Planetary Nebulae Parameters

\begin{tabular}{|c|c|c|c|c|}
\hline Parameter & NGC 2346 & NGC 6720 & NGC 7293 & Reference \\
\hline $\mathrm{H}_{2} 1-0 S(1)\left(\mathrm{ergs} \mathrm{cm}^{-2} \mathrm{~s}^{-1} \mathrm{sr}^{-1}\right) \ldots \ldots$ & $(6 \pm 2) \times 10^{-5}$ & $(1.5 \pm 0.5) \times 10^{-4}$ & $(6 \pm 1) \times 10^{-5}$ & $1,2,3$ \\
\hline 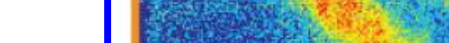 & $2500 \pm 500$ & $3000_{-1700}^{+2500}$ & $19,000_{-8000}^{+11,000}$ & 1,2 \\
\hline$D(\mathrm{pc}) \ldots \ldots$ & 800 & $500 \pm 200$ & $200 \pm 20$ & $1,4,5$ \\
\hline$v_{\exp }\left(\mathrm{km} \mathrm{s}^{-1}\right) \ldots \ldots \ldots \ldots \ldots \ldots \ldots \ldots \ldots \ldots \ldots \ldots \ldots$ & 25 & 27 & 20 & $1,6,7$ \\
\hline$r_{\text {in }}(\operatorname{arcsec}) \ldots \ldots \ldots \ldots \ldots$ & 15 & $25-35$ & $300-500$ & $1,2,3$ \\
\hline$n\left(\mathrm{~cm}^{-3}\right)$ & $7 \times 10^{3}$ & 500 & 60 & $1,3,8$ \\
\hline$\ldots \ldots \ldots \ldots \ldots \ldots \ldots \ldots$ & 0.7 & 0.69 & 0.93 & $1,9,10$ \\
\hline$L_{*}\left(L_{\odot}\right) \ldots \ldots \ldots \ldots \ldots \ldots \ldots \ldots \ldots \ldots$ & 250 & 120 & 100 & $1,3,11$ \\
\hline 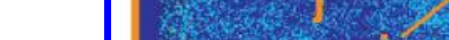 & $4.6 \times 10^{-4}$ & $5.9 \times 10^{-4}$ & $4.6 \times 10^{-4}$ & $1,8,10$ \\
\hline$\ldots \ldots \ldots \ldots \ldots \ldots \ldots \ldots$ & $5.3 \times 10^{-4}$ & $6.5 \times 10^{-4}$ & $4 \times 10^{-4}$ & $1,8,10$ \\
\hline $\mathrm{He} / \mathrm{H}$. & 0.13 & 0.12 & 0.12 & $1,8,10$ \\
\hline
\end{tabular}

References. - (1) Vicini et al. 1999; (2) this work; (3) Speck et al. 2002; (4) Pier et al. 1993; (5) Harris et al. 1996; (6) Bachiller et al. 1989; (7) Young et al. 1999; (8) Kwitter \& Henry 1998; (9) Galli et al. 1997; (10) Henry, Kwitter, \& Dufour 1999; (11) Pottasch 1984. 
increase on a timescale of a few hundred years. The Helix Nebula is the oldest and has an $\mathrm{H}_{2} 1-0 \mathrm{~S}(1)$ surface brightness lower than the Ring, following the predicted drop in surface brightness with age. The $\mathrm{H}_{2}$ surface brightness of the Helix Nebula is higher than the evolved NGC 2346 model because the core mass of the Helix is larger than NGC 2346's core mass. In the models of NH98, larger core mass PNe maintain higher $\mathrm{H}_{2}$ 1-0 $S(1)$ surface brightnesses to much older ages than the lower core mass PNe. The error bars for the surface brightnesses in Figure 3 reflect the range of observed brightnesses in these sources. The error bars for the dynamical ages, which are quite uncertain, take into account the uncertainty in distances and inner radii (see Table 1).

Figure 3 shows that the three nebulae, NGC 2346, the Ring Nebula, and the Helix Nebula, exhibit different stages of PN evolution in terms of their $\mathrm{H}_{2}$ emission. This is consistent with the evolutionary scheme suggested by O'Dell et al. (2002), who observed an evolution in the nature of the molecular knots, such that the Ring Nebula is a middle-aged PN, while the Helix is an old PN.

\section{CONCLUSIONS}

We have presented a new high-resolution molecular hydrogen image of the Ring Nebula, covering only the main, optically bright ring. The high spatial resolution of this image allows us to see the extremely clumpy nature of the molecular material in the Ring Nebula. The distribution of the molecular emission closely follows that of the ionized gas.

Comparing the high-resolution $\mathrm{H}_{2}$ image with the Hubble Heritage image of the Ring, it is clear that the dark clumps seen by HST match the locations of clumpy $\mathrm{H}_{2}$ emission, sug- gesting that these clumps are similar to the cometary knots seen in the Helix Nebula. Indeed, the overall morphology of the $\mathrm{H}_{2}$ emission image for the Ring Nebula is remarkably similar to that of the $\mathrm{H}_{2}$ emission for the Helix Nebula.

Comparison of the observed $\mathrm{H}_{2}$ surface brightnesses for NGC 2346, the Ring Nebula, and the Helix Nebula with the time-dependent models of the expected $\mathrm{H}_{2}$ emission shows that it is consistent with $\mathrm{H}_{2}$ excitation in PDRs. The $\mathrm{H}_{2}$ is thermally excited in PDRs at the surface of the dense molecular clumps, where the molecular gas meets the ionized gas. The comparison with time-dependent models also suggests an evolutionary progression in the $\mathrm{H}_{2}$ emission, which coincides with that seen in the ionized gas images of the clumpy structures of PNe. This evolution is mirrored in the morphology of the knots seen in $\mathrm{H}_{2}$ emission, which are more isolated in the Helix Nebula than in the Ring Nebula, suggesting that a longer evolution has allowed more erosion of the molecular gas.

On the basis of the models, the $\mathrm{H}_{2}$ emission from the Ring Nebula is expected to get twice as bright on the timescale of a few hundred years.

We would like to thank Antonella Natta for the model data included in Figure 3 and David Hollenbach for details about the models. Howard Bond is thanked for his Hubble Heritage image of the Ring Nebula. Charles Corson is thanked for making NIRIM work on WIYN. We are grateful to Danielle Moser and Luke Pyzowski for their help with reducing the standard stars. We would also like to thank Joel Kastner for access to his $\mathrm{H}_{2}$ image of the Ring Nebula and for helpful correspondence. Ian Gatley and Jim Kaler are also thanked for constructive comments and conversations. A. K. S. was supported by NASA JPL 961504 and NASA STI 7898.02-96A. A. K. S. and M. M. were supported by NSF CAREER award AST 97-33697.

\section{REFERENCES}

Acker, A., Marcout, J., Ochsenbein, F., Stenholm, B., \& Tylenda, R. 1992, Strasbourg-ESO Catalogue of Galactic Planetary Nebulae (Garching: ESO)

Bachiller, R., Bujarrabal, V., Martin-Pintado, J., \& Gomez-Gonzalez, J. 1989, A\&A, 218, 252

$\rightarrow$ Balick, B., Gonzalez, G., Frank, J., \& Jacoby, G. 1992, ApJ, 392, 582

$\rightarrow$ Beckwith, S., Gatley, I., \& Persson, S. E. 1978, ApJ, 219, L33

Bond, H., et al. 1998, BAAS, 30, 1276

Bryce, M., Balick, B., \& Meaburn, J. 1994, MNRAS, 266, 721

$\rightarrow$ Capriotti, E. 1971, ApJ, 166, 563

Dyson, J. E., Hartquist, T. W., Pettini, M., \& Smith, L. J. 1989, MNRAS, 241, 625

$\rightarrow$ Elias, J. H., Frogel, J. A., Matthews, K., \& Neugebauer, G. 1982, AJ, 87,1029

$\rightarrow$ Galli, D., Stanghellini, L., Tosi, M., \& Palla, F. 1997, ApJ, 477, 218 Gathier, R. 1987, A\&AS, 71, 245

$\rightarrow$ Greenhouse, M. A., Hayward, T. L., \& Thronson, H. A. 1988, ApJ, 332, 1092

Harris, H. C., Dahn, C. C., Monet, D. G., \& Pier, J. R. 1996, in IAU Symp. 180, Planetary Nebulae, ed. H. J. Habing \& H. J. G. L. M. Lamers (Dordrecht: Kluwer), 40 $\rightarrow$ Henry, R. B. C., Kwitter, K. B., \& Dufour, R. J. 1999, ApJ, 517, 782

Huggins, P. J., \& Healy, A. P. 1986, MNRAS, 220, P33

$\rightarrow \longrightarrow$. 1989, ApJ, 346, 201

$\rightarrow$ Huggins, P. J., et al. 2002, ApJ, 573, L55

$\rightarrow$ Hunt, L. K., Mannucci, F., Testi, L., Migliorini, S., Stanga, R. M., Baffa, C., Lisi, F., \& Vanzi, L. 1998, AJ, 115, 2594

$\rightarrow$ Kastner, J. H., Gatley, I., Merrill, K. M., Probst, R., \& Weintraub, D. 1994, ApJ, 421, 600

$\rightarrow$ Kastner, J. H., Weintraub, D., Gatley, I., Merrill, K. M., \& Probst, R. 1996, ApJ, 462, 777

$\rightarrow$ Kwitter, K. B., \& Henry R. B. C. 1998, ApJ, 493, 247

$\rightarrow$ Latter, W. B., Dayal, A., Bierging, J. H., Meakin, C., Hora, J. L., Kelly, D. M., \& Tielens, A. G. G. M. 2000, ApJ, 539, 783

$\rightarrow$ Latter, W. B., Kelly, D. M., Hora, J. L., \& Deutsch, L. K. 1995, ApJS, 100,159

$\rightarrow$ Meaburn, J., Clayton, C. A., Bryce, M., Walsh, J. R., Holloway, A. J., \& Steffen, W. 1998, MNRAS, 294, 201

$\rightarrow$ Meixner, M., Young Owl, R., \& Leach, R. W. 1999, PASP, 111, 997 Natta, A., \& Hollenbach, D. 1998, A\&A, 337, 517 (NH98)

$\rightarrow$ O'Dell, C. R., Balick, B., Hajian, A. R., Henney, W. J., \& Burkert, A. 2002, AJ, 123, 3329 
$\rightarrow$ O’Dell, C. R., \& Handron, K. D. 1996, AJ, 111, 1630

Pier, J. R., Harris, H. C., Dahn, C. C., \& Monet, D. G. 1993, in IAU Symp. 155, Planetary Nebulae, ed. R. Weinberger \& A. Acker (Dordrecht: Kluwer), 175

Pottasch, S. R. 1984, Planetary Nebulae: A Study of Late Stages of Stellar Evolution (Dordrecht: Reidel)

$\rightarrow$ Speck, A. K., Meixner, M., Fong, D., McCullough, P. R., Moser, D. E., \& Ueta, T. 2002, AJ, 123, 346
Vicini, B., Natta, A., Marconi, A., Testi, L., Hollenbach, D., \& Draine, B. T. 1999, A\&A, 342, 823

$\rightarrow$ Vishniac, E. T. 1994, ApJ, 428, 186

$\rightarrow$ Young, K., Cox, P., Huggins, P. J., Forveille, T., \& Bachiller, R. 1999, ApJ, 522, 387

$\rightarrow$ Zhang, C. Y. 1993, ApJ, 410, 239

$\rightarrow$ Zuckerman, B., \& Gatley, I. 1988, ApJ, 324, 501 